\title{
Calculation of bone graft volume using 3D reconstruction system
}

\author{
José-Luis Calvo-Guirado ${ }^{1}$, José-Eduardo Maté-Sánchez ${ }^{2}$ Rafael Delgado-Ruiz ${ }^{2}$, Maria-Piedad Ramírez- \\ Fernández $^{3}$
}

\footnotetext{
${ }^{1}$ Doctor of Dentistry. Senior Lecturer in Integrated Adult Dentistry, University of Murcia

${ }^{2}$ Doctor of Dentistry. Associate Lecturer in Dental Pathology and Therapy, University of Murcia

${ }^{3}$ Doctor of Dentistry. In private practice
}

Correspondence:

Paseo Alfonso XIII $N^{\circ} 12,9^{\circ} \mathrm{B}$

30201. Cartagena. Murcia. Spain

jmsodont@hotmail.com

Received: 23/03/2010

Accepted: 26/08/2010



\begin{abstract}
Objectives: To obtain bone "bioreplicas" for determining precisely the amount of biomaterial required for bone regeneration procedure. Study design: A case-control comparison with a total sample size of 20 cases, 10 control and 10 test samples. "Bioreplicas" were generated from helical CAT scans with $0.5 \mathrm{~mm}$ slices, without 3D reconstruction or image filters. Bone defects in premolar and molar areas were treated with titanium mesh and xenograft (MP3) combined with resorbable carriers. Time taken to carry out procedures with and without the use of "bioreplicas" (in minutes), discrepancies between the grafts performed with and without "bioreplicas" and bone defects $(\mathrm{mm})$, and postoperative complications were registered. Results: No significant differences were recorded for measurements of width and length of bone defect between patient bone and the biological models. Conclusions: The use of "bioreplicas" obtained by rapid prototyping is effective in treatment planning.
\end{abstract}

Key words: Stereolithography, biological models, xenograft.

\section{Introduction}

Determining the volume of bone deficit for purposes of oral and maxillofacial surgery is an important area for research (1). Prior knowledge of bone quantity, volume and position can facilitate surgical procedures and reduce treatment morbidity in cases of autologous bone grafts, as well as facilitating treatment planning in cases of xenografts (2).

Advances in tomography image technology and reconstruction software have made it possible to generate three-dimensional anatomical structures based on high resolution computerized axial tomography (CAT scans)
(3). The clinical benefits of these biomodels have been identified both for diagnosis and pre-operative planning in fields that include orthopedic surgery, neurosurgery and craniofacial surgery (4).

Studies such as Arias-Irimia demonstrate the effectiveness of computer imaging techniques for the calculation of three-dimensional bone graft volumes, ending in the simplicity and effectiveness of the techniques (5).

According to D'Urso (6), "biomodel is a generic term that has been coined to describe replications of the morphology of a solid biological structure." On this basis, a biomodel reproduces the geometry or morphology 
of a biological structure and may exist as a computergenerated entity or as a solid physical model (7). The term bioreplica refers to an entity that reproduces the morphology of a biological structure maintaining a 1:1 proportion with respect to clinical reality. This may be either a digital image or a physical structure (8). These three-dimensional models generated by computerized tomography allow the faithful reproduction of anatomical structures. The opportunity to visualize the unique structures of the individual patient prior to surgery is of great help for planning, pre-visualizing and simulating treatment and is especially important in complex or critical cases (9). In cases presenting craniofacial anomalies, dysplasias and developmental defects, 3D models are particularly useful for designing surgical incisions and resections. This will result in time saved and it is possible to model the graft prior to intervention by making surgical templates (10). Furthermore, the opportunity to differentiate radiological densities in the digital image obtained makes it possible to visualize two adjoining or limiting structures by digitally coloring different areas (11). Composite powder molding is a rapid prototyping technology which consists of casting a physical object in three dimensions by the agglutination of a succession of powder layers. Once the model has been taken in powder, an infiltrating product must be applied to confer the model's final physical properties such as hardness, temperature tolerance etc.

The precision obtained with this process is described in terms of two values:

Layer thickness: The thickness of the slices in which the 3D model is divided.

Resolution: The mold resolution of each of these slices. For the purposes of this study these values are:

- Layer thickness: $0.085 \mathrm{~mm}$.

- Resolution: 600 x 540 dpi.

Composite powder components:

- Plaster (50-95\%)

- Silica $(<1 \%)$

- Polyvinyl (2-20\%)

- Carbohydrate (1-20\%)

- Sulfate salt (1-20\%)

Standard composite infiltrator:

-Formula with urethane base

Alternative composite infiltrators:

-Beta-methoxyethyl Cyanoacrylate (80-100\%)

-Epoxy resin.

-Fabrication Process:

Radiology Procedure: To produce a high quality bioreplica, computerized axial tomography (CAT scan) should be carried out using a medical helical-cone beam machine without contrast filters or reconstruction of the register obtained, given that images will be reconstructed from an unprocessed DICOM format. The thinner the slices scanned, the more precise the model will be,
0.5-1.5 mm being the minimum slice thickness.

3D Model Generation: A digital three-dimensional model of the bone to be reproduced is obtained from the CAT scan register.

Molding the Model Matrix: The 3D model is then divided in a succession of two-dimensional layers or slices that are then molded one by one with an agglutinating agent in a composite powder tank. This operation may take several hours depending on the complexity of the model shape. The object produced is extremely fragile so that it must remain in the composite powder for at least twenty-four hours before being moved in order to keep it in one piece. Model Cleaning: Once the model has been removed from the composite powder it must stand for at least an hour before starting the cleaning procedure. This consists of removing any powder untouched by agglutinating agent still clinging to the model using compressed air. Infiltration: The final step in the process consists of infiltrating the model with one of the infiltration materials listed above, selected according to the requirements of the finished product. These materials penetrate and bind, solidify and harden the composite powder body. Sterilization: Biomodels produced using this technology can be sterilized chemically using, for example, ethylene oxide. Autoclave sterilization is not recommended

The three-dimensional molding system based on molding in layers brings a series of advantages compared to traditional stereolithography, which is based on a subtraction model to obtain an object, whilst composite powder molding builds in layers and then refines them, achieving greater precision.

The aim of our study was to obtain, from helical CAT scans, volumetric biomodels of bone for precisely determining the biomaterial required for bone regeneration procedure, this in order to validate the use of bioreplicas for calculating bone volumes prior to planning bone regeneration surgery.

\section{Materials and Methods}

A comparative study was made with a total sample size of twenty cases, ten in the control group (without biomodels) and ten in the test group.

The twenty cases all required bone graft implants as a response to moderate alveolar atrophy; ten of these were treated by conventional means (filling in the defect with progressive material increments until completely restored) and the other ten made use of biomodels. In the cases with biomodels, helical CAT scans were carried out in $0.05 \mathrm{~mm}$ slices without three-dimensional reconstruction or image filtering. Images were stored in DICOM format and processed using algorithm reconstruction software specific to the molding system, which used a composite similar to natural bone. The molding technique was carried out with a layer width 

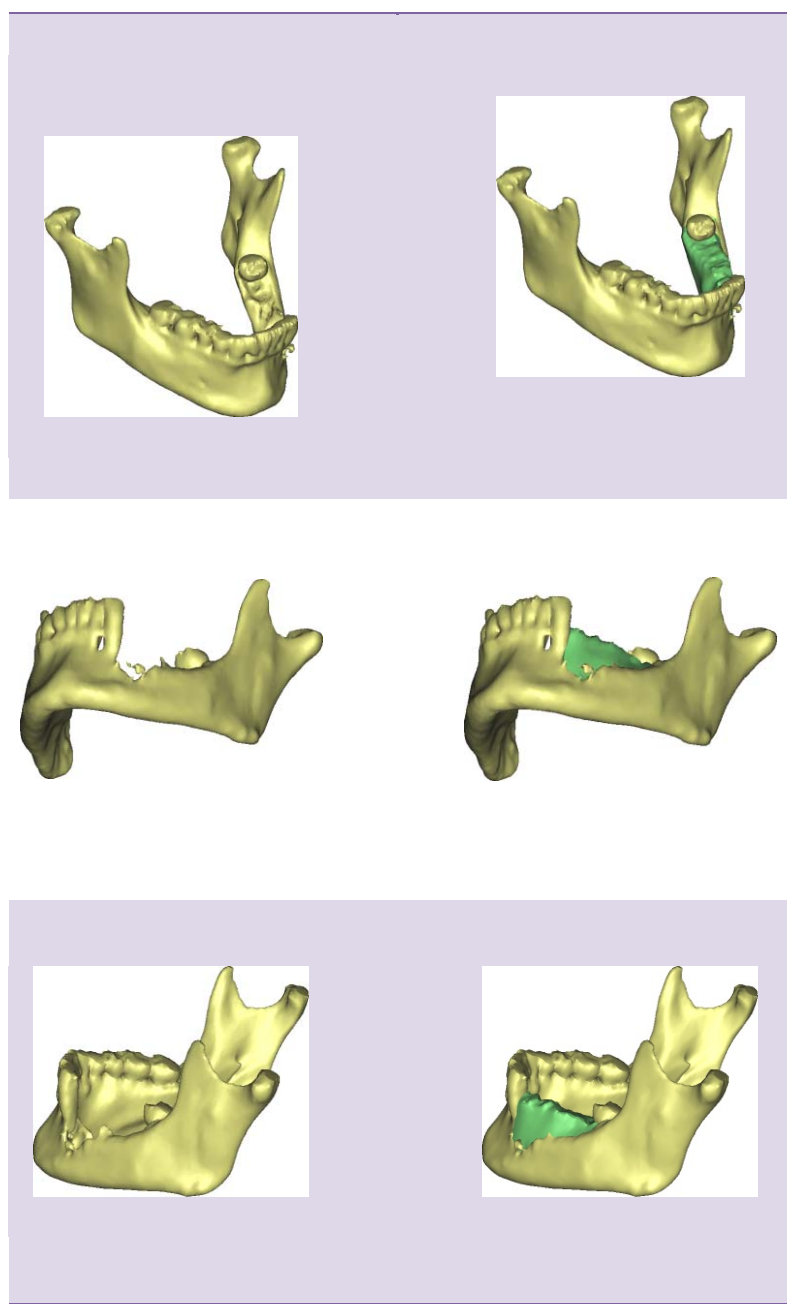

Fig. 1. Graft design. Shows the planning and design process of the future graft from which a biomodel is taken for the graft's physical fabrication. of $0.085 \mathrm{~mm}$, to guarantee the precision of the sample reproduction (Fig. 1).

All patients suffered unilateral bone defects in molar and premolar areas, indicating treatment with titanium mesh and xenografts combined with a resorbable carrier material (Fig. 2). The bone replacement material was MP3 (Osteobiol $\AA$, Osteogenos, Madrid, Spain) a mixture of cortical and spongy bone of porcine origin in form of radiopaque hydroxyapatite granules and with a granulometry of $600-1000 \mu \mathrm{m}$ and containing collagen. Titanium mesh was fitted using standard screws. In order to calculate the volume of bone deficit the CAT scans stored in unprocessed DICOM format underwent digital image reconstruction at $0.5 \mathrm{~mm}$; the bone deficit zone was reconstructed by extrapolating the contra-lateral zone and creating a mirror image. Image processing was carried out using OsiriX v. 3.6.1 for Mac image analysis software (Fig. 3). The reconstructed zone

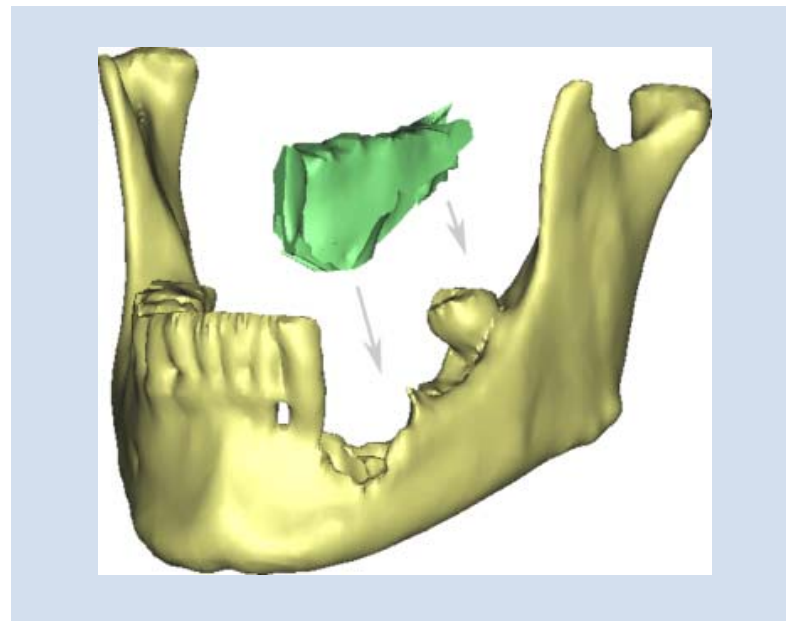

Fig. 2. Location. Shows the planned location of the future graft.
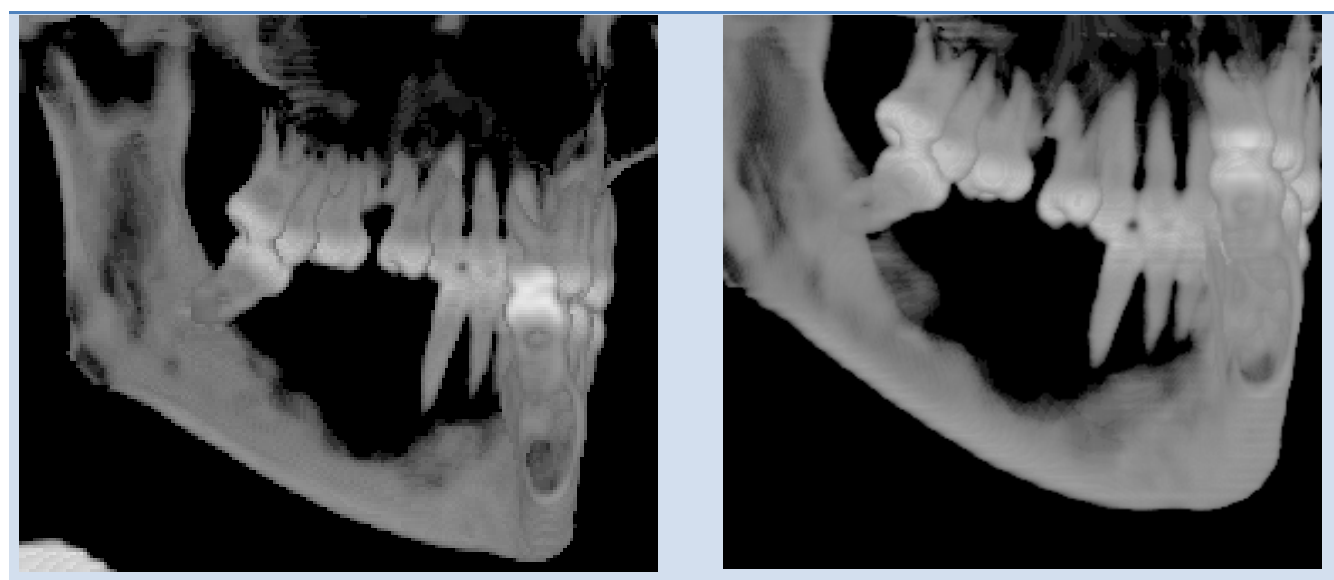

Fig. 3. Case at start of treatment. Shows the clinical case prior to surgery. Reconstructed CT image. 
stands out in relief in relation to the reception area facilitating visualization and ensuring adaptation of the form. Three-dimensional composite powder molding of the graft models was carried out, calculating the total volume required to reconstruct each defect area; these calculations were carried out using measurement data obtained with the CAT scans. The models generated were sterilized so that they could then be used to assist the surgical intervention. The graft blocks themselves could then be shaped making use of these volume calculations as well as the bioreplicas. Treatments both with and without the use of bioreplicas were timed (in minutes); discrepancies between the grafts preformed from bioreplicas and bone defects were evaluated (in millimeters) and post-operatory complications occurring with either technique (mesh exposure and/or wound dehiscence) were noted in two examinations held fifteen and thirty days after surgery. The data obtained was compiled as average \pm standard deviation and processed using SPSS 17.0 statistical analysis software in order to compare averages. Evaluations were made at the moment of surgery and post-operatory complications were noted at fifteen and thirty days afterwards.

\section{Results}

The results obtained in the present study show that, in measurements taken from CAT scans, there were no significant differences between patients' bone defects and bioreplicas, with a signification of $95 \%$ for $\mathrm{p}<0.05$. There were no significant differences between measurements taken directly from the patients of the length and width of bone defects and the biomodels in any of the twenty cases. Adaptation of bone grafts was seen to be very precise showing only minimal discrepancies, none of which were greater than those considered acceptable with conventional techniques and none were greater than $1 \mathrm{~mm}$ (average value \pm standard deviation). The distance from the grafts' edges, whose forms were based on bioreplicas, to the edges of the reception areas were evaluated, these being less than $1.5 \pm 0.8 \mathrm{~mm}$. Time taken to carry out the procedure was considerably reduced using bioreplicas, cutting time by $45 \pm 13$ minutes in comparison with conventional techniques using grafts in material increments fixed with titanium mesh and screws. There were no significant differences between the test group and control group arising from post-surgical complications observed at the post-operatory fifteen and thirty day examinations. In this way, no increase in suture dehiscence occurred, no was the titanium mesh exposed, events that would have compromised the final outcome.

\section{Discussion}

The use of anatomical bone replicas for planning surgical treatment is justified by the fact that they facilitate the creation of blocks of bone graft and reduce their morbidity (12). The precision of these replicas gives a real idea of the particular features of each case prior to surgery and avoids the affectation of surrounding anatomical structures which might otherwise be damaged. Furthermore, the use of bioreplicas makes a 'rehearsal' of the surgical intervention possible without involving the patient. Other advantages offered by the creation of bone replicas include the opportunity to save time by allowing effective planning prior to surgery and an opportunity to become familiar with the area in which the operation will take place both in terms of the reception area and the graft itself. In this way, he showed that the use of bioreplicas in planning orthognatic surgery reduced work time by one hour compared to conventional techniques. It was also seen to reduce mandible reconstruction time by two hours (13). These data would agree with our own on the basis that clearer anatomical knowledge of the area and familiarity with the technique will make it much easier to obtain a donor graft (14).

3D composite cast models provide highly precise information about the anatomical structure to be treated and the graft site, whether this be as donor or receiver (15). The precision of biomodels has been described by such authors who showed that when fabricating splints and titanium prothesis on the basis of data taken either from patients or from biomodels, both sources achieved identical results and no significant differences were observed (16). Biomodels can be used to deal with complex biomaterial and graft structures which will be entirely compatible thanks to the models' capacity to reproduce structures precisely and, once sterilized, their capacity as a potent tool both for planning and surgery itself. In this way, the use of biomodels to create titanium structures for mandible reconstruction procedures in patients with mandibular defects, achieving excellent results in terms of reproducibility and sample precision (17). These biomodels showed very similar measurements to the CAT source data, showing a difference of less than $0.29 \mathrm{~mm}$ between the average measurements obtained. The use of bone bioreplicas generated by rapid prototype composite molding is an effective tool for planning and a valid and reliable resource for surgery. 18 The precision of bioreplicas make it possible to obtain a graft from a donor site accurately and, by doing so, reduce the time taken to carry out surgical procedures and also reduce morbidity. In the case of xenografts, the fact that bioreplicas can be sterilized makes them useful for producing graft inserts of the correct size and shape. 


\section{References}

1. Lal K, White GS, Morea DN, Wright RF. Use of stereolithographic templates for surgical and prosthodontic implant planning and placement. Part I. The concept. J Prosthodont. 2006;15:51-8.

2. Curcio R, Perin GL, Chilvarquer I, Borri ML, Ajzen S. Use of models in surgical predictability of oral rehabilitations. Acta Cir Bras. 2007;22:387-95.

3. Bouyssié JF, Bouyssié S, Sharrock P, Duran D. Stereolithographic models derived from $\mathrm{x}$-ray computed tomography. Reproduction accuracy. Surg Radiol Anat. 1997;19:193-9.

4. Barker TM, Earwaker WJ, Frost N, Wakeley G. Integration of 3-D medical imaging and rapid prototyping to create stereolithographic models. Australas Phys Eng Sci Med. 1993;16:79-85.

5. Arias-Irimia O, Barona-Dorado C, Martínez-Rodríguez N, Ortega-Aranegui R, Martínez-González JM. Pre-operative evaluation of the volume of bone graft in sinus lifts by means of CompuDent. Med Oral Patol Oral Cir Bucal. 2010;15:e512-6.

6. D’Urso PS, Barker TM, Earwaker WJ, Bruce LJ, Atkinson RL, Lanigan MW, et al. Stereolithographic biomodelling in craniomaxillofacial surgery: a prospective trial. J Craniomaxillofac Surg. 1999;27:30-7.

7. Lohfeld S, Barron V, McHugh PE. Biomodels of bone: a review. Ann Biomed Eng. 2005;33:1295-311.

8. Izatt MT, Thorpe PL, Thompson RG, D'Urso PS, Adam CJ, Earwaker JW, et al. The use of physical biomodelling in complex spinal surgery. Eur Spine J. 2007;16:1507-18.

9. Spencer KR, Sizeland A, Taylor GI, Wiesenfeld D. The use of titanium mandibular reconstruction plates in patients with oral cancer. Int J Oral Maxillofac Surg. 1999;28:288-90.

10. Gateno J, Xia J, Teichgraeber JF, Rosen A, Hultgren B, Vadnais $\mathrm{T}$. The precision of computer-generated surgical splints. J Oral Maxillofac Surg. 2003;61:814-7.

11. Cunningham LL Jr, Madsen MJ, Peterson G. Stereolithographic modeling technology applied to tumor resection. J Oral Maxillofac Surg. 2005;63:873-8.

12. Kernan BT, Wimsatt JA 3rd. Use of a stereolithography model for accurate, preoperative adaptation of a reconstruction plate. J Oral Maxillofac Surg. 2000;58:349-51.

13. Erickson DM, Chance D, Schmitt S, Mathis J. An opinion survey of reported benefits from the use of stereolithographic models. J Oral Maxillofac Surg. $1999 ; 57: 1040-3$.

14. Cohen A, Laviv A, Berman P, Nashef R, Abu-Tair J. Mandibular reconstruction using stereolithographic 3-dimensional printing modeling technology. Oral Surg Oral Med Oral Pathol Oral Radiol Endod. 2009;108:661-6.

15. Singare S, Dichen L, Bingheng L, Yanpu L, Zhenyu G, Yaxiong L. Design and fabrication of custom mandible titanium tray based on rapid prototyping. Med Eng Phys. 2004;26:671-6.

16. Yi Z, Jian-Guo Z, Guang-Yan Y, Ling L, Fu-Yun Z, Guo-Cheng $Z$. Reconstruction plates to bridge mandibular defects: a clinical and experimental investigation in biomechanical aspects. Int J Oral Maxillofac Surg. 1999;28:445-50.

17. Sannomiya EK, Silva JV, Brito AA, Saez DM, Angelieri F, Dalben Gda S. Surgical planning for resection of an ameloblastoma and reconstruction of the mandible using a selective laser sintering 3D biomodel. Oral Surg Oral Med Oral Pathol Oral Radiol Endod. 2008;106:e36-40.

\section{Acknowledgments}

Perdentex, Elche (Alicante), Spain. 